\title{
Entorno virtual y su impacto en el aprendizaje en estudiantes de
}

\section{ciencias de la salud.}

\author{
Virtual environment and its impact on learning in health science students.
}

Ambiente virtual e seu impacto na aprendizagem de estudantes de ciências da saúde.

\section{ARTÍCULO GENERAL}

Lusin Antonio Ponce

Contreras

https://orcid.org/0000-0002-

5523-8134

lponcec@unmsm.edu.pe

Universidad Nacional Mayor de San Marcos
Misael Jefferson Fajardo Quispe

https://orcid.org/ 0000-0002-

6543-223X

mfajardoq@unmsm.edu.pe

Universidad Nacional Mayor de San Marcos
Luisa Lucía Quispe Valladares https://orcid.org/ 0000-0002-2957$\underline{7722}$

lquispeva@unmsm.edu.pe

Universidad Nacional Mayor de San Marcos
Deysi Díaz Ramos

http://Orcid.org/0000-00017573-2335

ddiazra@unmsm.edu.pe

Universidad Nacional Mayor de San Marcos

Recibido 12 de Setiembre 2021 | Arbitrado y aceptado 12 de Setiembre 2021 | Publicado en 12 de Octubre 2021

\section{RESUMEN}

Esta investigación tiene como objetivo general analizar el entorno virtual y su impacto en el aprendizaje en estudiantes de ciencias de la salud. La metodología aplicada se basó en un diseño bibliográfico con un tipo de investigación documental. Los resultados se basaron en análisis sobre la importancia que tienen los entornos virtuales, la formación académica en ciencias de la salud en los entornos virtuales y como desarrollar las prácticas de ciencias de la salud en estos medios. Como conclusión se puede determinar que los estudiantes tienen una buena formación académica a través de los diversos entornos virtuales porque tienen la capacidad de buscar la información, comprobarla y generar el conocimiento necesario para desarrollarla en la vida profesional; esto potenciado con las practicas de simulación que permite verificar parámetros que no se pueden determinar en la práctica presencial, así como también permite conocer nuevas técnicas diagnósticas y tratamientos antes de ser probados, con el fin único de mejorar la calidad de vida de los pacientes.

Palabras claves: ciencia de la salud, formación académica, entorno virtual, simulación.

\section{ABSTRACT}

The general objective of this research is to analyze the virtual environment and its impact on learning in health sciences students. The applied methodology was based on a bibliographic design with a type of documentary research. The results were based on analyzes of the importance of virtual environments, academic training in health sciences in virtual environments, and how to develop health science practices in these media. As a conclusion, it can be determined that students have a good academic training through the various virtual environments because they have the ability to search for information, check it and generate the necessary knowledge to develop it in professional life; This is enhanced with simulation practices that allows to verify parameters that cannot be determined in face-to-face practice, as well as to learn about new diagnostic techniques and treatments before being tested, with the sole purpose of improving the quality of life of patients.

Keywords: health science, academic training, virtual environment, simulation

\section{RESUMO}

O objetivo geral desta pesquisa é analisar o ambiente virtual e seu impacto na aprendizagem de estudantes de ciências da saúde. A metodologia aplicada baseou-se em um desenho bibliográfico com modalidade de pesquisa documental. Os resultados foram baseados em análises sobre a importância dos ambientes virtuais, a formação acadêmica em ciências da saúde em ambientes virtuais e como desenvolver práticas de ciências da saúde nessas mídias. Como conclusão, pode-se constatar que os alunos têm uma boa formação acadêmica através dos diversos ambientes virtuais, pois possuem a capacidade de buscar informações, verificá-las e gerar o conhecimento necessário para desenvolvê-las na vida profissional; Isso é aprimorado com práticas de simulação que permitem verificar parâmetros que não podem ser determinados na prática presencial, bem como aprender sobre novas técnicas diagnósticas e tratamentos antes de serem testados, com o único objetivo de melhorar a qualidade de vida dos pacientes. .

Palavras-chave: ciências da saúde, formação acadêmica, ambiente virtual, simulação 


\section{Introducción}

Un aspecto importante en el desarrollo evolutivo de las sociedades es su capacidad de aprendizaje y de generación de conocimientos, lo cual ha permitido solventar los más grandes problemas como también ayudar a satisfacer sus más implícitas necesidades. Ha sido la formación a través de la educación que ha permitido un gran avance, generando conocimiento por medio de profesionales de diversas áreas. La educación se ha convertido en el pilar de todas las sociedades y es que su avance y desarrolle es directamente proporcional al desarrollo de la educación de la población.

En el mismo sentido, la educación ha formado parte de las infinitas ciencias que ha determinado el hombre. Ciencias que permiten la demostración de muchas teóricas a través de la realización de métodos y técnicas que permiten convalidarlas. Es así como la práctica dentro de la educación es un factor clave para la comprensión de los diversos fenómenos que puedan pasar en una determinada disciplina. Pero esta situación depende de la capacidad y receptividad de la persona que recibe esa información. Según Keefe (1988) los estilos de aprendizaje se definen como los rasgos cognitivos, afectivos y fisiológicos que sirven como indicadores relativamente estables, de cómo los discentes perciben, interaccionan y responden a sus ambientes de aprendizaje (Muñoz, RosetyRodríguez, \& Rosety-Plaza, 2003).

Del mismo modo, el desarrollo cognitivo de la persona que recibe la información debe procesarla y transfórmala en un conocimiento que se ha generado gracias a múltiples variables percibidas. Un parámetro fundamental para poder generar el conocimiento es la guía que le brinda la persona del saber, la cual intenta transferirle la sabiduría específica. Para este caso, en la asesoría del profesor o instructor de la educación. La orientación debe satisfacerse a través de la relación tutorial, como un medio para consolidar la formación integral del estudiante y, por medio de programas de apoyo especializados que deben funcionar paralela y solidariamente a la docencia y la tutoría (Psicología educativa) (García, 2002).

Esta simbiosis entre el docente y el alumno servirá para desarrollar el profesional del mañana. Este tendrá las cualidades pertinentes para solucionar o mejorar las diversas problemáticas que se le presentan a la sociedad. El docente brindará las herramientas y el estudiante con su capacidad cognitiva permitirá analizarlas, evaluarlas y desarrollarlas en los momentos adecuados. Es por ello, que la educación universitaria 
Lusin Antonio Ponce Contreras, Misael Jefferson Fajardo Quispe, Luisa Lucía Quispe Valladares, Deysi Díaz Ramos

logra formar profesionales e investigadores competentes, críticos, creativos y reflexivos; pero ante todo con profesionalismo y conciencia moral (Pinilla-Roa, 2012).

En el mismo modo, este logro de la educación universitaria es producto de una serie de factores que inciden directamente en el desarrollo de las habilidades tanto pedagógicas, por parte de los docentes, como de las necesidades de lograr los conocimientos por parte del estudiante. Estos dos actores forman al nuevo profesional integro, capaz y eficiente. Todo esto pasa naturalmente por desarrollar la capacidad para aprender autónomamente, pues la formación continuada personal es la única garantía contra la obsolescencia profesional, por lo que debe estimularse, y nunca coartarse, la creatividad, el espíritu emprendedor y la iniciativa (Baños \& Pérez, 2005).

Toda esa situación debe permitir enfocarse en una de las ciencias más importantes en el desarrollo y bienestar de la sociedad como es la generación de profesionales en el área de la salud. Este desarrollo pedagógico y de compromiso docente-estudiante debe ser mucho mayor porque los resultados se observan en la mejora de la calidad de vida de los pacientes que sufren de múltiples patologías. Por esta situación, la salud pública y la promoción de la salud demandarán un enfoque amplio basado en el desarrollo humano integral, en el cual la salud, además de ser un objetivo en sí mismo, sea un medio para la vida y para el desarrollo social (Cardona \& Franco, 2005).

La formación académica en esta área es más compleja que el de otras ciencias por que incluye ámbitos que requieren de métodos y técnicas más complejas debido a que analizan la anatomía del cuerpo humano en búsqueda de poder ayudar a solventar los síntomas de las enfermedades. Es decir, cada solución es inédita para cada situaciónproblema, por ejemplo, un caso clínico o de laboratorio, no es un modelo o fórmula predeterminadas (Pinilla-Roa, 2012). De esta situación, se plantea que la estructura académica en esta área depende de dos modelos bases, uno biomédico y el otro tradicional.

El modelo biomédico se basa en el establecimiento de relaciones de imposición de significados, donde uno de los actores (el profesional de la salud) tiene el conocimiento "verdadero" y el otro, el paciente, posee un saber no reconocido por el primero. Así que la educación, en el escenario de la salud, se configura como proceso impositivo doble: por el modelo biomédico y por el modelo tradicional de educación. (Díaz, y otros, 2010, pág. 222) 
Lusin Antonio Ponce Contreras, Misael Jefferson Fajardo Quispe, Luisa Lucía Quispe Valladares, Deysi Díaz Ramos

De la sinergia de estos dos modelos existen factores que inciden en la formación del profesional de la salud. El apoyo institucional y sus cuadros, el trabajo consciente y consagrado de los docentes, la dedicación al estudio de los estudiantes y la disponibilidad de recursos materiales para la docencia, son elementos determinantes para alcanzar una formación de calidad (Carreño de Celis, Salgado González, Fernández Oliva, \& Alonso Pardo, 2009).

Por consiguiente, el factor que incide en el desarrollo de estos profesionales porque modifica los demás factores es el desarrollo y capacitación de los docentes. Estudios han determinado que, por ejemplo, la Deficiencia Metodológica del profesorado aparece en todos los casos como la principal circunstancia del contexto académico generadora de estrés en los estudiantes (Casuso Holgado, 2011).

De esta situación, es necesario un acompañamiento a los docentes en la formación metodológica y pedagógica. Además, se suma la necesidad del desarrollo de habilidades (competencias) comunicativas y emocionales (solidaridad, compasión, humanidad), que soporten e inspiren la relación con el paciente y al mismo tiempo involucren al estudiante (Sáenz-Lozada, Cárdenas-Muñoz, \& Rojas-Soto, 2010).

La preparación permanente e integral del docente de tercer nivel en las ciencias de la salud constituye un eje fundamental en la formación del estudiante y en la transformación de la sociedad. La misma se sustenta en adquisición por estos de las habilidades del lenguaje, la comunicación, del pensamiento crítico, ético y del conocimiento de un idioma extranjero, así como del manejo de las tecnologías de la información y las comunicaciones, lo que les permitirá integrar las funciones sustantivas de la universidad: docencia, investigación y vinculación, guiadas estas por la investigación formativa. (González, Molina, Peña, Herrera, \& Larramendi, 2018, pág. 37)

Es por ello, que es necesario aplicar una serie de mecanismos que permitan una mejor interacción entre el docente y el estudiante. Las actividades que pueden fomentar el dominio de numerosas competencias de origen transversal durante la formación universitaria son: aprendizaje basado en problemas, trabajos de investigación, programas de postgrado y formación en competencias (Baños \& Pérez, 2005; Pinilla, 2011). En la Tabla 1 se describe las características del aprendizaje basado en problemas y en la Tabla 2 de desarrolla las características de formación pedagógica basada en competencias.

Tabla 1. 
Lusin Antonio Ponce Contreras, Misael Jefferson Fajardo Quispe, Luisa Lucía Quispe Valladares, Deysi Díaz Ramos

Características del aprendizaje basado en problemas.

Implica procesos de:

- Exploración del entorno.

- Reconocimiento de la situación problema del caso.

- Ejecución de actividades de resolución.

- Integración de las ciencias básicas y las clínicas.

- Lectura crítica de la literatura relacionada.

- Reestructuración de concepciones previas.

- Trabajo en equipo colaborativo.

- Síntesis de posibles respuestas.

- Desarrollo de competencias clínicas, de comunicación e investigación.

Fuente: (Pinilla, 2011)

Tabla 2.

Aspectos y características de la formación pedagógica basada en competencias.

\begin{tabular}{ll}
\hline \multicolumn{1}{c}{ Aspectos } & \multicolumn{1}{c}{ Características } \\
\hline Jerarquiza & Contenidos \\
\hline Meta & $\begin{array}{l}\text { Desarrollo personal, aprender a pensar, aprender a } \\
\text { aprender }\end{array}$ \\
\hline Enfoque del aprendizaje & Profundo, significativo para la comprensión, \\
\hline Enfoque del conocimiento & En construcción y renovación permanente \\
\hline Fuentes de conocimiento & Docente, alumno, textos \\
\hline Papel del alumno & $\begin{array}{l}\text { Activo, autónomo, participativo, agente de inda- } \\
\text { gación e investigación }\end{array}$ \\
\hline $\begin{array}{l}\text { Estrategias didácticas } \\
\text { (metodología) }\end{array}$ & $\begin{array}{l}\text { Acorde a estructuras cognitivas del estudiante. } \\
\text { El profesor coordina el plan con los intereses del } \\
\text { estudiante, se basa en problemas que relacionan el } \\
\text { conocimiento cientíico con el cotidiano. }\end{array}$ \\
\hline Tipo de aprendizajes & $\begin{array}{l}\text { Horizontal, bidireccional, en equipo colaborativo } \\
\text { para construir conocimiento por proceso participa- } \\
\text { tivo }\end{array}$ \\
\hline Relación maestro-alumno & $\begin{array}{l}\text { Interdisciplinar y transdisciplinar } \\
\text { sentido, asocia lo nuevo con lo que ya sabe, aprende } \\
\text { lo que le motiva y propone al profesor temas y } \\
\text { preguntas a resolver }\end{array}$ \\
\hline $\begin{array}{l}\text { Proceso permanente; abarca la evaluación diagnós- } \\
\text { dica, formativa y terminal para evaluar el grado de } \\
\text { desarrollo de competencias profesionales }\end{array}$ \\
\hline
\end{tabular}

Fuente: (Pinilla, 2011)

También, el docente puede fomentar el desarrollo profesional y ético del estudiante a través del acompañamiento con el contacto directo con los pacientes. La 
Lusin Antonio Ponce Contreras, Misael Jefferson Fajardo Quispe, Luisa Lucía Quispe Valladares, Deysi Díaz Ramos

tutoría y guía de los docentes permiten formar análisis sobre las patologías presentes en tiempo real. Es necesario promover el trabajo comunitario como eje articulador de la docencia, la investigación y la extensión, pues se percibió como importante poseer un alto sentido de compromiso social, producto de haberse dejado sensibilizar por la comunidad, sus problemas y sus intereses (Díaz, y otros, 2010).

Por otra parte, es importante poder resaltar que en la actualidad hay un factor clave en desarrollo académico de los estudiantes y es el uso de las tecnologías de la información y comunicación (TIC). A través de ambientes virtuales se pueden desarrollar las diversas áreas pedagógicas y científicas que permiten fomentar el desarrollo de la lógica en las diversas áreas de las ciencias de la salud. Estos entornos virtuales se pueden apoyar en plataformas que puedan describir con exactitud los diversos contenidos de los programas académicos, por medio de la utilización de simulación, con el complemento de las redes sociales como factor de debate y generador de ideas.

Sin duda existe un gran avance en el acercamiento del conocimiento sobre las ciencias de la salud, solo quedando como amenazas y debilidades los recursos tecnológicos que deben poseer los involucrados en el desarrollo académico, es decir los docentes y estudiantes, así como los programas prácticos tan fundamentales en la comprensión y desarrollo lógico de los profesionales.

Por lo tanto, esta investigación tiene como objetivo general analizar el entorno virtual y su impacto en el aprendizaje en estudiantes de ciencias de la salud. La metodología aplicada se basó en un diseño bibliográfico con un tipo de investigación documental.

\section{Metodología}

La investigación se basó en un diseño bibliográfico de tipo documental. El diseño se fundamenta en la revisión sistemática, rigurosa y profunda de material documental de cualquier clase, donde se efectúa un proceso de abstracción científica, generalizando sobre la base de lo fundamental, partiendo de forma ordenada y con objetivos precisos (Palella Stracuzzi \& Martins Pestana, 2010).

La investigación documental se concreta exclusivamente en la recopilación de información de diversas fuentes, con el objeto de organizarla describirla e interpretarla de acuerdo con ciertos procedimientos que garanticen confiabilidad y objetividad en la presentación de los resultados (Palella Stracuzzi \& Martins Pestana, 2010). Las fuentes 
Lusin Antonio Ponce Contreras, Misael Jefferson Fajardo Quispe, Luisa Lucía Quispe Valladares, Deysi Díaz Ramos

se obtuvieron de textos, documentos y artículos científicos publicados disponibles en la web.

Los objetivos de esta investigación se basaron en describir la importancia de la formación académica en ciencias de la salud, analizar la importancia de las TIC, evaluar el entorno virtual de las asignaturas de ciencias de la salud y determinar si las materias prácticas de la carrera de ciencias de la salud es factible desarrollarla en los entornos virtuales.

\section{Resultados y discusión}

\section{La importancia de los entornos virtuales.}

El desarrollo del aprendizaje no ocurre por mera casualidad. Debe existir un compromiso del individuo y su búsqueda del conocimiento, una búsqueda del desarrollo personas. "Conocer" es una actividad compleja, que implica razón y emoción, cerebro y corazón, que comprende la construcción de representaciones mentales que "imponen" orden y coherencia sobre la información y la experiencia humana (Carneiro, 2009).

En este sentido, la búsqueda del conocimiento en la actualidad se ha visto favorecida por el desarrollo de las TIC, la computadora personal y el internet juega un papel importante en dicho crecimiento. De este último, ha permitido acercar, en tiempo real, la información produciendo una generación de conocimiento casi al instante. El uso de internet como método de aprendizaje es tan efectivo como los métodos tradicionales, permite una accesibilidad mucho mayor, y se muestra, en algunos aspectos como en la adquisición de conocimientos, superior a los métodos tradicionales de enseñanza (de Toledo \& Cáceres, 2008).

El ordenador y el internet se han transformado en instrumentos que permiten que el individuo pueda generar más conocimiento debido a la búsqueda incesante de información, mas no dan garantía del mismo sino existe un compromiso entre los individuos involucrados. Es por ello, necesario contextualizar este término. Las TIC son el conjunto de tecnologías que permiten el acceso, producción, tratamiento y comunicación de información presentada en diferentes códigos (Ortí, 2011).

En la perspectiva del aprendizaje, la utilización de las TIC tiene grandes ventajas: interés, motivación, interacción, continua actividad intelectual, desarrollo de la 
iniciativa, mayor comunicación entre profesores, alumnos y alumnas, aprendizaje cooperativo, alto grado de interdisciplinariedad, alfabetización digital y audiovisual, desarrollo de habilidades de búsqueda y selección de información, mayor contacto con los estudiantes, actualización profesional. (López \& Constante, 2018, pág. 29)

En este mismo contexto, las TIC de manera genera tiene un funcionamiento dentro de la generación de conocimiento, tal como se describe en la Figura 1.

FUNCIONES DE LAS TIC EN EDUCACIÓN
- Medio de expresión: escribir, dibujar, presentaciones, webs..
- Canal de comunicación, colaboración e intercambio.
- Instrumento para procesar la información.
- Fuente abierta de información (mass media, self media)
- Instrumento para la gestión administrativa y tutorial.
- Herramienta de diagnóstico y rehabilitación.
- Medio didáctico: informa, entrena, guia aprendizaje, motiva
- Generador de nuevos escenarios formativos
- Medio lúdico y para el desarrollo cognitivo.
- Contenido curricular: conocimientos, competencias

Figura 1. Las funciones de las Tic en la Educación. Fuente: (Graells, 2013)

Sin embargo, al detallar estas funciones se percibe que las TIC no generan por si misma el conocimiento sino más bien sirven de puente o guía para tal fin. Es decir, las TIC son utilizadas más en los procesos mismos de preparación de los procesos de enseñanza (administración, gestión, preparación de las clases, búsquedas, etc.), que en el proceso mismo de enseñanza (Quintero, 2009). Aunado, a que se podría afirmar que las TIC no pueden impactar positivamente la calidad del aprendizaje de los estudiantes si en la filosofía del diseño de enseñanza-aprendizaje no se aprovechan plenamente las posibilidades que ofrecen dichas tecnologías (Rodríguez-Izquierdo, 2011).

Por lo cual, un instrumento que puede permitir una mayor accesibilidad a la información para que los individuos que interactúan en ella generen los conocimientos pertinentes sobre el fin de ese medio, son los entornos virtuales. Según RiverosHernández et al (2017), es un espacio educativo usualmente disponible en internet, que integra recursos educativos, promueve la interactividad de los estudiantes y brinda actividades de aprendizaje estructuradas (Gutiérrez-Rodríguez, 2018). Del mismo modo,

El Dr. Rafael Emilio Bello Díaz (2005) llama a los entornos virtuales para el aprendizaje "aulas sin paredes" y afirma que es un espacio social virtual, cuyo mejor exponente actual es la Internet, no es presencial, sino representacional, no es proximal, sino distal, no es sincrónico, sino multicrónico, y no se basa en recintos espaciales con 
Lusin Antonio Ponce Contreras, Misael Jefferson Fajardo Quispe, Luisa Lucía Quispe Valladares, Deysi Díaz Ramos

interior, frontera y exterior, sino que depende de redes electrónicas cuyos nodos de interacción pueden estar diseminados por diversos países. (Delgado \& Solano, 2015, pág. 2)

Por lo cual, estos entornos virtuales permiten con mayor efectividad la interacción entre los protagonistas del aula de clase en un ambiente tradicional. El desarrollo del conocimiento es más amplio por la gama de información que maneja el internet, aunado a la interactividad que pueda tener el individuo. Es por ello, que en la Tabla 3 se muestran las ventajas de esta nueva forma pedagógica de la educación.

\section{Tabla 3.}

Ventajas de los entornos virtuales.

El efecto de atracción social para los alumnos que implica un acercamiento del aprendizaje informal con el formal

La sencillez y fomento de la comunicación entre el docente y los estudiantes, en ambas direcciones, al estar todos en un mismo espacio.

Presenta alto grado de libertad en la organización de los usuarios, para afrontar los objetivos de los proyectos-retos en los que participan, facilitado por la existencia de diversos tipos de expresión de la naturaleza del contenido.

Es integrable con entornos físicos, como demuestran los altos niveles de participación registrados en la plataforma virtual durante los días de trabajo en el aula física.

Permite el aprendizaje autónomo y continuo a través del ciclo problema-retopropuesta-evaluación, que realiza el grupo de usuarios participantes de manera colectiva.

Fuente: (Rodríguez Gallego \& López Martínez, 2013; Osuna-Pérez \& AbarcaÁlvarez, 2013)

En contexto, los entornos virtuales son espacios que permiten una interacción efectiva entre los participantes, más sin embargo es un compromiso de estos mismos, sobre manera del docente que orienta sobre el conocimiento que se debe adquirir. Por lo que, existe una necesidad de cambio en cuanto a los modelos pedagógicos tradicionales, donde el papel de los docentes debería ser el de liderar la incorporación de nuevas estrategias formativas al aula, lo cual permitiría mejorar en gran medida la educación (Gutiérrez-Rodríguez, 2018). Es por ello, que

No se puede pretender únicamente trasladar a la plataforma virtual los materiales y actividades que se utilizaban en el aula presencial, sino que se debe desarrollar apoyos 
Lusin Antonio Ponce Contreras, Misael Jefferson Fajardo Quispe, Luisa Lucía Quispe Valladares, Deysi Díaz Ramos

multimedia que integren audio, imagen, texto y, si es posible, que sean interactivas, así como actividades que promuevan el aprendizaje colaborativo mediante herramientas lúdicas, trabajos cuya finalidad sea el desarrollo del pensamiento crítico y el debate, debido a que es necesario atraer al estudiante a este entorno, darle las herramientas y guías necesarias para que desarrolle las actividades y alcance exitosamente las metas propuestas. (Delgado \& Solano, 2015, pág. 18)

\section{Entorno virtual en la educación para ciencias de la salud.}

Una de las ciencias más importantes es la de medicina debido a su complejidad en la resolución de los casos clínicos porque depende de la sintomatología de cada individuo. Es por ello, que en la educación tradicional exige un gran compromiso de los estudiantes y docentes porque el profesional debe garantizar una mejor calidad de vida a los pacientes. De tal modo, que la información debe ser adquirida para que los sujetos la transformen en el conocimiento, no solo académico sino personal.

De igual manera, las ciencias de la salud han presentado fallas en las áreas pedagógicas tradicionales. Esto permite que se fomenten nuevos espacios o instrumento pedagógicos que ayuden a solventar esos espacios vacíos generados por la educación presencial. Los factores se detallan en la Tabla 4.

\section{Tabla 4.}

Factores que afectan el desarrollo del conocimiento en ciencias de la salud en la educación tradicional.

Los hospitales de tercer nivel han venido transformándose en hospitales de atención ambulatoria o de corta estancia y sólo los pacientes más críticos tienen estancias prolongadas. Esto conlleva a que los estudiantes no tengan acceso a un espectro completo de experiencias educativas, teniendo menos oportunidad de observar y analizar pacientes con diversas condiciones, enfermedades, signos y síntomas clínicos

Aunque han estado siempre presentes, las consideraciones sobre la seguridad y el bienestar de los pacientes, así como las implicaciones éticas y legales son cada vez más exigentes y obligan a replantear el método tradicional de adquisición de habilidades clínicas en hospitales de alta complejidad, basado en "ver uno, hacer uno, enseñar uno"

Confiar en los pacientes hospitalizados para adquirir habilidades clínicas, hace que la enseñanza pueda depender de la presentación de los casos que llegan al hospital, dejando por fuera otras situaciones clínicas importantes.

Estudiantes de pregrado entrenados en diferentes instituciones podrían diferir en 
Lusin Antonio Ponce Contreras, Misael Jefferson Fajardo Quispe, Luisa Lucía Quispe Valladares, Deysi Díaz Ramos

sus habilidades, destrezas o competencias ya que tendrían diversas oportunidades para las experiencias educativas.

Los escenarios donde se tratan pacientes críticos constituyen sitios donde se integran los conocimientos básicos con los clínicos y se ponen a prueba las habilidades y los conocimientos. Sin embargo, frecuentemente los estudiantes de pregrado son excluidos del manejo de dichos pacientes, sin considerar que en su ejercicio posterior se pueden enfrentar situaciones similares.

La reducción de oportunidades para la enseñanza tutorial personalizada en las actividades quirúrgicas, debido al aumento de los costos y al número de estudiantes, ha generado la necesidad de usar modelos, cadáveres y animales para replicar las situaciones clínicas y, más recientemente, al desarrollo de centros o laboratorios para desarrollar habilidades quirúrgicas

Las implicaciones personales, sociales y económicas de las complicaciones en los procedimientos invasivos o quirúrgicos

El desarrollo de nuevas técnicas quirúrgicas endoscópicas o mínimamente invasivas, que requieren destrezas diferentes a las de los procedimientos quirúrgicos abiertos, han limitado la práctica de los estudiantes de pregrado y de postgrado y generado la necesidad de entrenamientos adicionales.

Las restricciones que impone la atención en salud teniendo en cuenta indicadores de eficiencia, contención de costos y mejoramiento de la calidad. Esto ha implicado una limitación para la docencia directa con los pacientes institucionalizados y ambulatorios.

Los cambios en la percepción de los pacientes hacia los estudiantes de las ciencias de la salud. Anteriormente los pacientes reconocían al "Doctor(a) estudiante como el profesional en sí', apoyado por un(a) "profesor(a) eminente de medicina" que agregaba con su experiencia un beneficio adicional, haciendo que la situación de caridad se tornara en una ventaja en su favor. En el nuevo marco legal, el (la) estudiante se le percibe como "el (la) practicante", una persona inexperta y sin conocimiento quien, más que aportar por su cuidado, lo entorpece y le produce molestias y pérdidas de tiempo innecesarias.

La proliferación de facultades y el aumento del número de estudiantes frente a una disponibilidad restringida de sitios de enseñanza. Los pacientes se pueden sentir incomodados, invadidos y utilizados cuando son valorados en forma repetida, de manera individual o grupal, por razones educativas.

La necesidad de incluir en los programas de pregrado las técnicas de 
Lusin Antonio Ponce Contreras, Misael Jefferson Fajardo Quispe, Luisa Lucía Quispe Valladares, Deysi Díaz Ramos

reanimación básica y avanzada que constituyen un requisito indispensable para todo médico graduado.

La evaluación positiva por estudiantes y graduados que han participado en diversas experiencias con nuevas tecnologías educativas.

Fuente: (Ruiz-Parra, Ángel-Muller, \& Guevara, 2009)

Una de las herramientas que permite mejorar todos estos factores son los entornos virtuales. Este permite que se desarrolle más información que ayude a formar todos los conocimientos necesarios para desempeñar una labor eficiente. Para que se desarrolle de manera efectiva debe existir un acompañamiento de la universidad al docente con el fin de que reciba apoyo en su formación, ya no del área donde es especialista, sino en el dominio de las TIC. Este proceso de guía institucional se puede realizar a través de proyectos de investigación; talleres con la comisión de currículo; plan de capacitación docente y acompañamiento a los docentes para el montaje de las aulas virtuales (Henríquez Gabante, Veracoechea Frisneda, Papale Centofanti, \& Berrios Rivas, 2015).

Los resultados de esta formación han permitido poder delimitar las funciones que puedan tener los entornos virtuales en las enseñanzas de las ciencias de la salud. Estos fueron: a) evitar el desarrollo de los ambientes de aprendizaje virtuales desde una visión muy amplia y compleja, b) identificar los repositorios como herramientas útiles para reusar conocimiento y c) Desarrollar la estrategia didáctica mediante aplicaciones sencillas que puedan ser accedidas desde cualquier ambiente o plataforma (Miguel, Fernández, Montaño, \& Lucci, 2013).

Estas formaciones han permitido que los estudiantes mejoren en su rendimiento porque se consideran espacios más amigables, una ventana a la búsqueda infinita del conocimiento. en otras palabras, Este método es igual o más efectivo que los procedimientos tradicionales de aprendizaje, expresando ventajas, principalmente por las habilidades en el manejo de la información, la satisfacción de los estudiantes con la metodología y la facilidad en la actualización de los contenidos (Álvarez, 2009).

En este mismo sentido, son los estudiantes el verdadero jurado que dictará la sentencia sobre la capacidad e influencia de los entornes virtuales como herramienta didáctica en las áreas de ciencia de la salud. Éste se involucra activamente en su aprendizaje, al utilizar los recursos para mejorar o completar una tarea, movilizar estrategias de gestión de recursos con actuación sobre factores externos (ambiente) e 
Lusin Antonio Ponce Contreras, Misael Jefferson Fajardo Quispe, Luisa Lucía Quispe Valladares, Deysi Díaz Ramos internos (sí mismo) para potenciar el logro de sus metas u objetivos (Palacios, Urrizola, \& Melo, 2019).

\section{El entorno virtual y las prácticas de medicina.}

Una de las debilidades que se ha determinado en el desarrollo de asignaturas en los entornos virtuales es lo referente a la realización de las prácticas. En la educación tradicional la realización de estas ha permitido fortalecer los conocimientos y teorías impartidas en el aula de clase. La formación en ciencias de la salud se ha caracterizado en un desarrollo de conocimientos a través de las prácticas y contactos personales con los pacientes. Tal situación ha permitido el fortalecimiento profesional de los egresados de las escuelas de medicina.

Es aquí, donde entra el reto de las TIC para poder corroborar dichas características. Los entornos virtuales pueden solventar esta situación gracias a que se conforma como una ventana abierta para que se desarrollen todos los contenidos, tanto teóricos como prácticos, sobre las ciencias médicas. Para lograr este objetivo es necesario que el instrumento, en este caso el aula virtual cumple una serie de requisitos. En este sentido, es necesario atender a un conjunto de indicadores que garanticen la calidad del escenario instructivo y, más específicamente, de su interfaz gráfico. En la Tabla 5 se desarrolla los indicadores que son necesario para poder realizar una interfaz amigable para los estudiantes de ciencias de la salud.

\section{Tabla 5.}

indicadores para una interfaz amigable con los estudiantes de ciencias de la salud.

\section{INDICADORES CARACTERÍSTICAS}

- El diseño de las pantallas deberá ser agradable, presentando un interfaz atractivo, que contribuya a que el estudiante se sienta cómodo con el formato visual a través del cual se presenta la información.

- Se deberá emplear una gama cromática que facilite la

Diseño de interfaz percepción de los contenidos, evitando las estridencias que abocan a la ansiedad, y dificultan la lectura de la información.

- A lo largo de la asignatura no se deberá abusar en la presentación de los contenidos didácticos de imágenes, documentos textuales o sonoros excesivamente voluminosos, etc., porque ralentizan su presentación "on line" 
Lusin Antonio Ponce Contreras, Misael Jefferson Fajardo Quispe, Luisa Lucía Quispe Valladares, Deysi Díaz Ramos

- Al estudiante se le permitirá acceder en todo momento al menú, facilitando de esta manera la flexibilidad en la navegación.

- Los iconos han de ser fácilmente reconocibles para los

Iconos y mapas de discentes, de otro modo, se incrementaría la complejidad navegación cognitiva que podría suponer identificarlos.

- Los estudiantes deberían poder reconocer en todo momento donde pueden encontrar hiperenlaces, apelando a un color de fuente diferente o mediante imágenes animadas fácilmente reconocibles.

\section{Metáforas del}

entorno

Presentación de la información
- Se deberá asegurar que la metáfora empleada sea familiar para el discente, y su presentación sea suficientemente explícita.

- El tamaño y tipo de letra adoptado deberá facilitar la lectura de la información presentada en la pantalla. Deberá evitarse intercalar animaciones innecesarias y superfluas en la presentación del texto, ya que pueden dificultar la lectura o distraer del objetivo principal.

- Los textos se presentarán sin faltas ortográficas, con construcciones gramaticales adecuadas y simples, los cuales estarán dotados de un vocabulario apropiado y comprensible.

- Las imágenes incorporadas deberán adecuarse y ser coherentes con la información textual presentada. Y del mismo modo, los documentos sonoros deberán ser claros y nítidos, primando la pertinencia y la complementariedad entre éstos y el texto.

Fuente: (Moral Pérez \& Villalustre Martínez, 2009)

Un aspecto importante de estos entornos virtuales es la capacidad de poder adjuntar otras plataformas que permitan un mejor desarrollo de los conocimientos. Estas plataformas deben ser muy diversificadas que permitan la comprensión de los diferentes fenómenos que pueden ocurrir en la vida real. Un aspecto que se considera son las simulaciones, la cual se basa en la fidelidad y capacidades del instructor (docente).

Para Urra, Sandoval e Iribarren (2017), la fidelidad se refiere al grado normal de la realidad que es proyectada (la conducta de la simulación se relaciona a la conducta del mundo real) mientras que las capacidades del instructor se refieren al grado de 
Lusin Antonio Ponce Contreras, Misael Jefferson Fajardo Quispe, Luisa Lucía Quispe Valladares, Deysi Díaz Ramos

entrenamiento y habilidades que tiene el docente para incorporar la simulación al interior del aula de clase. (García, Aldás, \& Vaquero, 2017, pág. 229)

Por lo cual, la simulación se convierte en un factor clave para poder solventar las prácticas presenciales, además permiten solventar eventos más allá de los percibidos en un laboratorio. La educación basada en simulación es un método que demuestra altos niveles de confiabilidad en la enseñanza en áreas de la salud y se puede implementar con programas virtuales, maniquís y herramientas de ejecución clínica (Mantilla \& Santa, 2015).

Del mismo modo, es necesario comprender esta importante técnica para la formación académica de los estudiantes de ciencias de la salud. La simulación es la experiencia o ensayo que se realiza con la ayuda de un modelo, el cual es una representación idealizada de un sistema real (máquina simple o compleja), por lo que se pretende es simular el comportamiento de un sistema (Martínez, y otros, 2012). En un enfoque pedagógico la simulación es un término genérico para la representación artificial de un proceso de la vida real, que pretende lograr metas educativas por medio del aprendizaje de experiencias (Ruiz-Parra, Ángel-Muller, \& Guevara, 2009).

Del mismo modo, la simulación se ha convertido en el desarrollo de nuevas formas de conocimiento, porque permite indagar mucho más allá que al realizar el practicas presenciales. Indagar con los pacientes permite fomentar la incredulidad de estos últimos en los médicos. El desarrollo de nuevos diagnósticos y tratamientos puede probarse en simulaciones para evitar cometer los errores en personas y así acelerar su padecimiento. Es por ello, se ha utilizado la simulación como una metodología de enseñanza, con un currículo basado en competencias y cuyos objetivos sean claros, se puede mejorar significativamente la atención de nuestros pacientes, por parte de los nuevos estudiantes (Bustos-Álvarez, 2015).

Por lo tanto, los estudiantes pueden incrementar sus habilidades y destrezas en estos simuladores con el fin de otorgar una solución más efectiva que permita mejorar la calidad de vida de los pacientes.

Los estudiantes que participan en este método de enseñanza por simulación aumentan sus habilidades clínicas, conocimiento, seguridad y habilidad de comunicación en un equipo interdisciplinar. La implementación de ambientes simulados permite generar una política de seguridad al paciente minimizando la aparición de incidentes o eventos adversos y así incrementar su experiencia a nivel clínico y mejorar sus procesos de atención clínica. Se deben tener en cuenta los siguientes elementos para 
Lusin Antonio Ponce Contreras, Misael Jefferson Fajardo Quispe, Luisa Lucía Quispe Valladares, Deysi Díaz Ramos

la aplicación de educación basada en simulación: herramientas y programación de clase. (Mantilla \& Santa, 2015, pág. 76)

\section{Referencias bibliográficas}

Álvarez, B. F. (2009). Entornos virtuales como apoyo al aprendizaje de la anatomía en medicina. Revista Investigaciones Andina, 11(19), 94-106. Obtenido de https://revia.areandina.edu.co/index.php/IA/article/view/233/251

Baños, J. E., \& Pérez, J. (2005). Cómo fomentar las competencias transversales en los estudios de Ciencias de la Salud: una propuesta de actividades. Educación médica, 8(4), 40-49. Obtenido de http://scielo.isciii.es/pdf/edu/v8n4/05.pdf

Bustos-Álvarez, J. (2015). Aprendizaje basado en problemas y simulación clínica: aprendiendo por competencias en la educación en salud. Revista Hispanoamericana de Ciencias de la Salud, 1(2), 117-120. Obtenido de http://uhsalud.com/index.php/revhispano/article/view/59

Cardona, Á., \& Franco, Á. (2005). La salud pública como disciplina científica: fundamento para los programas de formación académica. Revista Facultad Nacional de Salud Pública, 23(2), 107-114. Obtenido de https://www.redalyc.org/pdf/120/12011106013.pdf

Carneiro, R. (2009). Las TIC y los nuevos paradigmas educativos: la transformación de la escuela en una sociedad que se transforma. En R. Carneiro, J. Toscano, \& T. Díaz, Los desafíos de las TIC para el cambio educativo (págs. 15-28). Madrid - España: Fundación Santillana.

Carreño de Celis, R., Salgado González, L., Fernández Oliva, B., \& Alonso Pardo, M. E. (2009). Factores que intervienen en el proceso de formación de los profesionales universitarios de la salud. Educación Médica Superior, 23(3), 82-95. Obtenido de http://scielo.sld.cu/pdf/ems/v23n3/ems08309.pdf

Casuso Holgado, M. J. (2011). Estudio del estrés, engagement y rendimiento académico en estudiantes universitarios de Ciencias de la Salud. Malaga, España: Tesis doctoral de la Facultad de Enfermería, Fisioterapia, Podología y Terapia Ocupacional de la Universidad de Malaga. Obtenido de https://riuma.uma.es/xmlui/bitstream/handle/10630/4926/TD\%20Maria\%20Jesus\%20C asuso $\% 20$ Holgado.pdf?sequence $=1 \&$ isAllowed $=y$ 
Lusin Antonio Ponce Contreras, Misael Jefferson Fajardo Quispe, Luisa Lucía Quispe Valladares, Deysi Díaz Ramos

de Toledo, B. J., \& Cáceres, J. R. (2008). Es tan efectivo el aprendizaje por Internet como el aprendizaje presencial? Evidencias en pediatría, 4(4). Obtenido de https://dialnet.unirioja.es/servlet/articulo?codigo=2769405

Delgado, M., \& Solano, A. (2015). Estrategias didácticas creativas en entornos virtuales para el aprendizaje. Actualidades Investigativas en Educación, 9(2), 1-22. Obtenido de http://euaem1.uaem.mx/bitstream/handle/123456789/1538/estrategias.pdf?sequence=1 \&isAllowed $=\mathrm{y}$

Díaz, P. A., Peñaranda, F., Cristancho, S., Caicedo, N., Garcés, M., Alzate, T., \& Gómez, S. N. (2010). Educación para la salud: perspectivas y experiencias de educación superior en ciencias de la salud, Medellín, Colombia. Revista Facultad Nacional de Salud Pública, 28(3), 221-230. Obtenido de https://www.redalyc.org/pdf/120/12018993002.pdf

García, B. L. (2002). Una aproximación al concepto de tutoría académica en el Centro Universitario de Ciencias de la Salud. Investigación en salud, 4(1), 1-12. Obtenido de https://www.redalyc.org/pdf/142/14240106.pdf

García, D. R., Aldás, M. E., \& Vaquero, M. E. (2017). Simulación clínica, una herramienta eficaz para el aprendizaje en ciencias de la salud. Revista Publicando, 4(13 (2)), 225-243. Obtenido de https://core.ac.uk/download/pdf/236643926.pdf

González, C. G., Molina, A. H., Peña, E. B., Herrera, Y. R., \& Larramendi, R. F. (2018). El docente de tercer nivel en las ciencias de la salud. Contexto ecuatoriano.

Educación Médica, 19(1), 34-38. Obtenido de https://www.sciencedirect.com/science/article/pii/S1575181316301255

Graells, P. M. (2013). Impacto de las TIC en la educación: funciones y limitaciones. 3C TIC, 2(1), 1-15.

Gutiérrez-Rodríguez, C. A. (2018). Fortalecimiento de las competencias de interpretación y solución de problemas mediante un entorno virtual de aprendizaje. Revista de Investigación, Desarrollo e Innovación, 8(2), 279-293. Obtenido de http://www.scielo.org.co/pdf/ridi/v8n2/2027-8306-ridi-8-02-00279.pdf

Henríquez Gabante, G., Veracoechea Frisneda, B., Papale Centofanti, J. F., \& Berrios Rivas, A. T. (2015). Modelo de capacitación docente para entornos virtuales de aprendizaje: Caso Decanato Ciencias de la Salud de la UCLA. RIED: Revista Iberoamericana de Educación a Distancia, 18(1), 67-90. Obtenido de http://espacio.uned.es/fez/eserv/bibliuned:revistaRied-2015-18-1-7030/Modelo.pdf 
Lusin Antonio Ponce Contreras, Misael Jefferson Fajardo Quispe, Luisa Lucía Quispe Valladares, Deysi Díaz Ramos

López, Y. A., \& Constante, G. F. (2018). Las TIC como herramienta de apoyo didáctico en el proceso de enseñanza-aprendizaje. Revista Científica Cátedra, 1(1), 2931.

Mantilla, J. I., \& Santa, J. M. (2015). Modelos de simulación clínica para la enseñanza de habilidades clínicas en ciencias de la salud. Movimiento Científico, 9(2), 70-79. Obtenido de https://dialnet.unirioja.es/servlet/articulo?codigo $=5524147$

Martínez, J. L., Prado, E. A., Ruiz, D. H., Caraballo, D. F., Mendoza, L. B., Hernández, T. G., \& Castro, M. (2012). Los simuladores y los modelos experimentales en el desarrollo de habilidades quirúrgicas en el proceso de enseñanza-aprendizaje de las Ciencias de la Salud. REDVET. Revista Electrónica de Veterinaria, 13(6), 1-23. Obtenido de https://www.redalyc.org/pdf/636/63624434013.pdf

Miguel, V., Fernández, M., Montaño, N., \& Lucci, F. (2013). Modelo para la enseñanza en ciencias, tecnología y salud en entornos virtuales. Cuadernos de la Escuela de Salud Pública, 1(86), 92-105. Obtenido de http://espacio.uned.es/fez/view/bibliuned:revistaRied-2015-18-1-7030

Moral Pérez, M. E., \& Villalustre Martínez, L. (2009). Evaluación de prácticas docentes universitarias desarrolladas en entornos virtuales. Pixel-Bit. Revista de Medios y Educación, 2009,(34), 151-163. Obtenido de https://idus.us.es/bitstream/handle/11441/22585/file_1.pdf?sequence=1\&isAllowed=y

Muñoz, F. O., Rosety-Rodríguez, M., \& Rosety-Plaza, M. (2003). Análisis de los estilos de aprendizaje predominantes entre los estudiantes de ciencias de la salud. Enfermería global, 2(2), 1-6. Obtenido de https://revistas.um.es/eglobal/article/view/619/645

Ortí, C. B. (2011). Las tecnologías de la información y comunicación (TIC). Univ. Val., Unidad Tecnol. Educ, (951), 1-7.

Osuna-Pérez, F., \& Abarca-Álvarez, F. J. (2013). Los nuevos roles en entornos educativos extendidos en Red. La experiencia de diseño de un entorno virtual de aprendizaje en Educación Superior. REDU. Revista de Docencia Universitaria, 11(2), 353-372. Obtenido de https://riunet.upv.es/handle/10251/140435

Palacios, M. S., Urrizola, C. G., \& Melo, B. C. (2019). Evaluación del curso virtual de Genética Humana en estudiantes universitarios de ciencias de la salud. . Revista Cubana de Educación Médica Superior, 33(3), 1-19. Obtenido de https://www.medigraphic.com/pdfs/educacion/cem-2019/cem193d.pdf 
Lusin Antonio Ponce Contreras, Misael Jefferson Fajardo Quispe, Luisa Lucía Quispe Valladares, Deysi Díaz Ramos

Pinilla, A. E. (2011). Modelos pedagógicos y formación de profesionales en el área de la salud. Acta Médica Colombiana, 36(4), 204-218. Obtenido de http://actamedicacolombiana.com/ojs/index.php/actamed/article/view/1451/427

Pinilla-Roa, A. E. (2012). Aproximación conceptual a las competencias profesionales en ciencias de la salud. Revista de Salud Pública, 14, 852-864. Obtenido de https://www.scielosp.org/pdf/rsap/2012.v14n5/852-864/es

Quintero, L. J. (2009). Las universidades apostando por las TIC: modelos y paradojas de cambio institucional. EDUTEC. Revista Electrónica de Tecnología Educativa, (28), 105., 1-14.

Rodríguez Gallego, M. R., \& López Martínez, A. (2013). Entorno virtual de aprendizaje compartido en Educación Superior. REDU: Revista de Docencia Universitaria, 1 (11), 411-428. Obtenido de https://idus.us.es/bitstream/handle/11441/17139/file_1.pdf?sequence=1

Rodríguez-Izquierdo, R. M. (2011). Repensar la relación entre las TIC y la enseñanza universitaria: problemas y soluciones. Profesorado. Revista de currículum y formación del profesorado. VOL. 15, $N^{o} 1,9-22$.

Ruiz-Parra, A. I., Ángel-Muller, E., \& Guevara, Ó. (2009). La simulación clínica y el aprendizaje virtual. Tecnologías complementarias para la educación médica. Revista de la Facultad de Medicina, 57(1), 67-79. Obtenido de https://revistas.unal.edu.co/index.php/revfacmed/article/view/14466/15319

Sáenz-Lozada, M. L., Cárdenas-Muñoz, M. L., \& Rojas-Soto, E. (2010). Efectos de la capacitación pedagógica en la práctica docente universitaria en salud. Revista de Salud Pública, 12, 425-433. Obtenido de https://www.scielosp.org/pdf/rsap/2010.v12n3/425-433/es 